\title{
Adhesion and Growth of Electrically Active Cortical Neurons on Polyethylenimine Patterns Microprinted Onto PEO-PPO-PEO Triblockcopolymer-Coated Hydrophobic Surfaces
}

\author{
Teun G. Ruardij*, Marc A. F. van den Boogaart, and Wim L. C. Rutten, Member, IEEE
}

\begin{abstract}
This paper describes the adhesion and growth of dissociated cortical neurons on chemically patterned surfaces over a time period of 30 days. The presence of neurons was demonstrated by measurement of spontaneous bioelectrical activity on a micropatterned multielectrode array. Chemical patterns were prepared with a combination of neurophobic layers of polyethylenoxide-polypropylenoxide-polyethylenoxide (PEO-PPO-PEO) triblockcopolymers adsorbed onto hydrophobic surfaces and neurophilic microprinted tracks of polyethylenimine (PEI). Results showed that commercially available PEO-PPO-PEO triblockcopolymers F108 and F127 (Synperonics, ICI) significantly reduced the adhesion of neuronal tissue when adsorbed on hydrophobic Polyimide (PI) and Fluorocarbon (FC) surfaces over a time period of eight days. In general, both F108- and F127-coated PI displayed equal or better neurophobic background properties after 30 days. Viability of neuronal tissue after 30 days on PEI microprinted F108- and F127-coated PI was comparable with relatively high viability factors between 0.9 and 1 (scale from 0 to 1). Summarizing, the strategy to combine the neurophobic adsorbed triblock-copolymers F108 and F127 onto hydrophobic surfaces with neurophilic microprinted PEI resulted in relatively long-term neuronal pattern preservation with high numbers of viable neurons present after 30 days.
\end{abstract}

Index Terms-Adhesion, cortical neurons, electrical activity, patterning, PEO-PPO-PEO, polyethylenimine, viability.

\section{INTRODUCTION}

C ONTROLLED integration of neuronal tissue with microchips requests combined expertise in neuronal cell culturing, chemical surface modification, and microelectronic engineering. Chang et al. [1], [2] published papers on the crossroad of these disciplines and demonstrated the possibility of bioelectrical recording from patterned neuronal networks using multielectrode arrays (MEAs) while Fromherz et al. [3] used topographically modified field effect transistors. This paper can be considered as a cross-fertilization of studies done

Manuscript received July 12, 2002; revised August 15, 2002. Asterisk indicates corresponding author.

*T. G. Ruardij is with the Faculty of Electrical Engineering, University of Twente, P.O. Box, 7500 AE, Enschede, The Netherlands (e-mail: t.g.ruardij@ el.utwente.nl).

M. A. F. van den Boogaart is with the Microsystems Laboratory, Ecole Polytechnique Federale de Lausanne (EPFL), CH-1015 Lausanne, Switzerland.

W. L. C. Rutten is with the Faculty of Electrical Engineering, University of Twente, 7500 AE, Enschede, The Netherlands.

Digital Object Identifier 10.1109/TNB.2002.806921 on bioelectronic recording from random neuronal networks [4]-[6] and fabrication of patterned neuronal networks on chemically or topographically modified surfaces [7]-[14].

The problem to create neuronal patterns with controlled two-dimensional designs and short live times ( $<10$ days) was solved years ago using a number of different techniques which all give more-or-less neuronal patterns [7]-[13]. However, preservation of the structure of patterned neuronal networks over longer periods of time ( $>15$ days) still remains a problem although Wheeler and coworkers have shown considerable progress in this field [14]. The problem of patterned network maintenance over prolonged time periods is twofold. The neuron-adhesive (neurophilic) part of the pattern should preferably be stable throughout the whole time period of the study while the nonadhesive (neurophobic) background part has to withstand the adsorption of cell-adhesive proteins, which in turn promote unwanted overgrowth with neuronal tissue. The source of the protein biofouling are the added proteins in the medium together with the endogenous proteins excreted in the medium during development of the neuronal network.

The type of interaction between neurons and the adhesive part of a chemical pattern can be nonspecific, specific, or both. The specific approaches with biomolecules [15], poly-peptides [14], or epitopes [17] are familiar neuron-adhesive choices but a common drawback is the hydrolysis of amide linkages in polypeptide backbones. The nonspecific electrostatic interaction between synthetic amide-linkage-free compounds like polyethylenimine (PEI) and negatively charged neuron-membranes is more promising from that perspective and demonstrated its applicability already in neuronal patterning studies using photoresist lift-off technology [17]. Application of the microcontact printing technique in combination with PEI as the printing substance is even more appealing as it opens the possibility to print relatively thick layers of PEI with multiple internal electrostatic attachment points.

The neuron-repellent behavior of materials is reported to take place according to two essentially different principles. One principle is based on the use of hydrophobic surfaces, which irreversibly adsorb nonadhesive proteins like albumin and subsequently prevent the adsorption of functional cell-adhesive proteins like laminin or fibronectin [17]. The second principle is based on the use of hydrophilic polyethylenoxide (PEO)-coated surfaces which can prevent the adsorption of all 
proteins presumably by steric hindrance [18]. Either way, both methods rely on the fact that functional cell-adhesive proteins can not adsorb onto the substratum surface and exert their important role in the adhesion process. The methods to fabricate PEO-coated surfaces can be divided in different subgroups in which covalent bonding of PEO chains [PEO also termed polyethylenglycol (PEG)] is one approach [14], [19]. Another approach is the presumed irreversible adsorption of combined hydrophilic-hydrophobic polyethylenoxide-polypropylenoxide (PEO-PPO-PEO) block-copolymers onto hydrophobic background materials [20], [21]. The advantage of covalent coupling of PEO chains to surfaces is the initial stability and, thus, prohibited displacement of PEO by proteins in solution. However, the chemical bonds between the PEO chains and the underlying substratum could be dissociated by the physiological surrounding in time after about 25 days [22]. The efficacy of simple adsorbed PEO-PPO-PEO triblockcopolymer layers as cell-repellent surfaces was demonstrated by Biran et al. [23] who used commercially available Pluronic ${ }^{\mathrm{TM}}$ F108 to inhibit adhesion of cortical astrocytes on polystyrene for up to two weeks in serum containing medium. Apparently the ability of astrocytes to remodel the underlying surface with adsorbing proteins was severely hampered by the presence of the F108. The cell-repelling feature of adsorbed PEO-PPO-PEO forms an interesting starting point for successful neuronal patterning studies over longer periods of time given the fact that neuron culturing usually takes place from more chemically defined media with less proteins that can interfere with the adsorbed PEO-PPO-PEO. In view of an application on MEAs with hydrophobic insulation layers, a practical advantage of adsorbed PEO-PPO-PEO layers is the fact that chemical modification procedures which could negatively interfere with other metal components like microelectrodes, are avoided. Therefore, adsorbed layers of PEO-PPO-PPO triblockcopolymers on hydrophobic surfaces will be tested as potential neurophobic candidates in patterning studies.

The aim of this paper is to study the adhesion, growth, and survival of cortical neurons on PEI patterns microprinted on PEO-PPO-PEO triblockcopolymer-coated surfaces. The efficacy of the commercially available PEO-PPO-PEO triblockcopolymers Synperonics F108 and F127 as neurophobic background surfaces will be evaluated over a time period of 30 days.

\section{MethodS}

\section{A. Cortical Neuron Isolation and Procedures}

Cerebral cortex from 1-day-old newborn rats was dissected out under sterile circumstances and cut into pieces of approximately $1 \mathrm{~mm}^{3}$. After collection, the tissue was trypsinized (0.25\% Trypsin/EDTA, Gibco) for $45 \mathrm{~min}$ in an incubator at $37^{\circ} \mathrm{C}$ at $5 \% \mathrm{CO}_{2}$ and subsequently treated with Soybean Trypsin Inhibitor (STI, $1 \mathrm{mg} / \mathrm{ml}$ ) and Desoxyribonuclease I (DNAse I, $1.1 \mathrm{unit} / \mathrm{ml}$ ). The dissociated tissue was spun down at $1200 \mathrm{rpm}$ during $5 \mathrm{~min}$ and resuspended in chemically defined medium R12 (DMEM/Ham's F12, Gibco) without serum [24]. Trypan blue stain $(0.4 \%)$ was used to discriminate and count living neurons in a Bürker chamber, prior to the sedimentation of the neurons onto the surfaces. Neurons were seeded onto the patterned structures with a plating density of 5000 living cells $/ \mathrm{mm}^{2}$. Cells were allowed to adhere onto the surfaces during a time period of $4 \mathrm{~h}$. Samples were rinsed with a $0.9 \% \mathrm{NaCl}$ solution to remove nonadherent cells.

\section{B. Microprinting of PEI With PDMS Stamps}

Sylgard 184 silicone (Mavom bv, The Netherlands) was mixed with the curing agent in a 10:1 ratio. Air bubbles were removed from the mixture by evacuation with a water jet pump. Collapse of air bubbles was promoted by following a cycle of evacuation and pressure release six times. A metal ring with an inner diameter of $17 \mathrm{~mm}$ (height $2 \mathrm{~mm}$ ) was placed around the central area of a mould (gold) with etched $15 \mu$ m-deep parallel tracks (width $15 \mu \mathrm{m}$ ) and a spacing distance between tracks of $145 \mu \mathrm{m}$. The ring was filled with the mixed silicone, covered with a 76-mm microscope slide and crosslinked at $120^{\circ} \mathrm{C}$ for $25 \mathrm{~min}$ on a heating plate. Finally, stamps were carefully removed from the mould and stored in plastic tubes until use. PEI (molecular weight is $6^{*} 10^{5}-10^{6}$, Fluka Zwijndrecht, The Netherlands) was applied as the printing substance (foreground) while several background substrates were tested. Stamps were made hydrophilic by exposing the PDMS to a gas flame shortly [25]. Then stamps were wetted with PEI $(10 \mathrm{mg} / \mathrm{ml})$ by manually pressing the stamp for $15 \mathrm{~s}$ into lint-free tissue (Hanotex BV, Joure, The Netherlands) soaked with PEI. To avoid the danger of capillary effects, evaporation of the residual water was visually checked until no changes were observed anymore. Finally, stamps with PEI were pressed onto the background materials using a pressure load of $0.55 \mathrm{~kg} / \mathrm{cm}^{2}$ for $15 \mathrm{~s}$.

\section{Bioelectrical Recording From Microprinted PEI Patterns}

The presence of neurons in postnatal tissue was demonstrated through extracellular recording of spontaneously formed bioelectrical activity in postnatal cultures (P1) using a home-built measurement system. In short, multielectrode areas (MEAs) were fabricated from $5 \times 5$-cm glass plates with gold deposited wires leading to 61 hexagonal ordered electrodes. MEAs were isolated with a sandwich layer of $\mathrm{SiO}_{2}-\mathrm{Si}_{3} \mathrm{~N}_{4}-\mathrm{SiO}_{2}$ (ONO) using a PECVD process. Electrode tips were deinsulated with a $\mathrm{SF}_{6}$ Reactive ion etching (RIE) technique and platinized to reduce the electrode impedance down to $200 \mathrm{k} \Omega$ at $1 \mathrm{kHz}$. The center-to-center distance between electrodes (diameter $10 \mu \mathrm{m}$ ) was $240 \mu \mathrm{m}$. The ONO sandwich layer was made hydrophobic by treatment with $0.1 \%$ (v/v) dichlorodimethylsilane (Merck, Darmstad, Germany) dissolved in Xylene for 10 min. Circles (diameter $100 \mu \mathrm{m}$ ) of PEI with a spacing distance in between of $90 \mu \mathrm{m}$ were microprinted onto the Dichlorodimethylsilane (DDS)-treated ONO layer. In order to measure bioelectrical activity, each MEA was placed in an aluminum mini-incubator providing contact between the gold leads and a selector. The selector connected 30 or 31 electrodes to 16 channels of an amplifier allowing switching between different electrode configurations. A Labview controlled data acquisition card was fed with the amplified signals and allowed scanning of the electrodes without data storage, continuous recording, or data storage of active periods only. In this last mode, a threshold for activity detection was computed from the root mean square 
value of all signals and a user defined signal-to-noise ratio of five. Each time bin of $10 \mathrm{~ms}$ with recorded activity was stored and analyzed with Matlab computer software.

\section{Scanning Electron Microscopy}

In order to prepare substrata for scanning electron microscopy, the substrata were immersed in $6.8 \%$ sucrose in $0.1 \mathrm{M}$ cacodylate (Cac.) buffer ( $\mathrm{pH}$ 7.4) to remove nonbound proteins and salt. Subsequently, cells were fixed with $2 \%$ glutaraldehyde in $0.1 \mathrm{M} \mathrm{Cac}$. buffer and stored in the fixative at $4^{\circ} \mathrm{C}$.

Cells were then washed with $6.8 \%$ sucrose in $0.1 \mathrm{M}$ Cac. buffer and post fixed with $1 \%$ osmiumtetroxide in $0.1 \mathrm{M} \mathrm{Cac}$. during $3 \mathrm{~h}$ at $4{ }^{\circ} \mathrm{C}$. Subsequently, cells were washes with $6.8 \%$ sucrose in $0.1 \mathrm{M} \mathrm{Cac}$. during $10 \mathrm{~min}$. and with water three times $10 \mathrm{~min}$. Thereafter, cells were dehydrated in an ethanol-water series of $30 \%, 50 \%$ and $70 \%$ ethanol, each step taking $10 \mathrm{~min}$. followed by three times $20 \mathrm{~min}$. in $100 \%$ ethanol. Finally, gold was sputter-coated to a thickness of $10 \mathrm{~nm}$. Samples were examined with a JEOL 6300 scanning electron microscope.

\section{E. Fabrication Background Materials}

1) Polyimide (PI): PI (Probimide 7510, Arch Chemicals N.V., Zwijndrecht, Belgium) was spin-coated (4000 rpm, $30 \mathrm{~s}$ ) onto $25-\mathrm{cm}^{2}$ glass plates (Glaverbel, Belgium). PI was diluted in n-methyl pyrolidon $(1: 1 \mathrm{v} / \mathrm{v})$, dried on a hot plate $\left(120^{\circ} \mathrm{C}\right.$, $5 \mathrm{~min}$ ), exposed to UV-light and baked $\left(300^{\circ} \mathrm{C}, 90 \mathrm{~min}\right)$. Then plates were cut into square pieces of approximately $2.6 \mathrm{~cm}^{2}$.

2) Plasma-Coated Fluorocarbon (FC): In an RIE system, spin-coated PI samples were treated with an etching $\mathrm{CHF}_{3} / \mathrm{O}_{2}$ plasma (25 sccm $\mathrm{CHF}_{3}, 5 \mathrm{sccm} \mathrm{O}, 150$ mtorr, and $\left.2.1^{*} 10^{-1} \mathrm{~W} / \mathrm{cm}^{2}\right)$ for $20 \mathrm{~s}$, a depositing $\mathrm{CHF}_{3}$ plasma $(25 \mathrm{sccm}$ $\mathrm{CHF}_{3}, 150 \mathrm{mtorr}$, and $2.1 * 10^{-1} \mathrm{~W} / \mathrm{cm}^{2}$ ) for $40 \mathrm{~s}$ and a final depositing $\mathrm{CHF}_{3}$ plasma treatment at $1.2^{*} 10^{-1} \mathrm{~W} / \mathrm{cm}^{2}$ for 8 min.

3) Synperonics F108 and F127: The triblockcopolymers Synperonics F108 $\left(\mathrm{EO}_{127}-\mathrm{PO}_{48}-\mathrm{EO}_{127}\right.$; ICI, Holland BV, Rozenburg) and F127 $\left(\mathrm{EO}_{95}-\mathrm{PO}_{62}-\mathrm{EO}_{95}\right.$; ICI, Holland BV, Rozenburg) were dissolved in $0.1 \mathrm{M}$ phosphate buffered saline (PBS) $(1 \% \mathrm{w} / \mathrm{w})$ and adsorbed onto PI- and Plasma-coated FC samples over a time period of $24 \mathrm{~h}$. Subsequently, samples were rinsed twice with sterile water (Aqua Purificata, Bufa BV, Uitgeest, The Netherlands). Surfaces were dried by aspiration of residual water with a glass pipette connected to a vacuum pump.

\section{F. Water Contact Angle Measurements}

Static water contact angles were measured with the sessile drop method (G40, Kruess, Germany) on the background materials under investigation. Stability of the F108- and F127-coated surfaces under physiological conditions was checked by measurements of the contact angles before and after storage in $0.1 \mathrm{M}$ PBS for 30 days. Samples were rinsed twice with sterile water and dried by aspiration of residual water with a glass pipette connected to a vacuum pump.

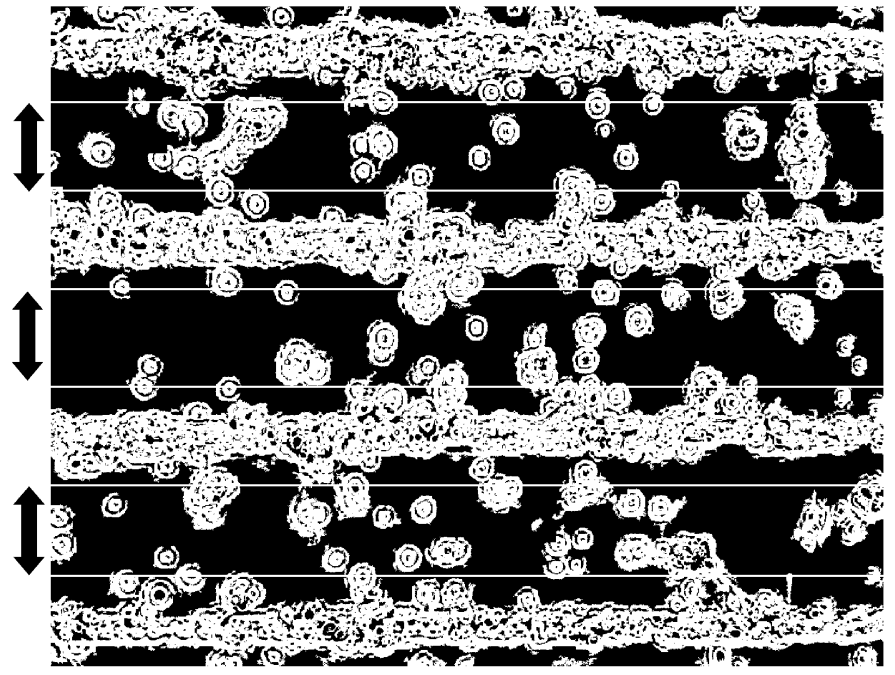

Fig. 1. Matlab processed image with adhering cortical neurons on microprinted multiple parallel PEI tracks. Only the adhesion on the background areas, indicated by the block arrows, was investigated.

\section{G. Quantification of Background Adhesion}

Microphotographs were taken on two separate subsections of each pattern after $1,4,8,15$, and 30 days with a digital photocamera (AxioCam HR, Carl Zeiss, Germany) attached to an inverted phase contrast microscope (Nikon Diaphot-TMD, Tokyo, Japan). Each time laps procedure was done on three different samples. Bitmap images were stored with 1030-1300 pixel resolution prior to image processing in Matlab (Version R11.1, The Mathworks Inc., Natick, MA). Calculation of the neuronal adhesion onto the background materials was assessed by a tresholding procedure which took into account possible disturbing nonuniform illumination effects that are usually present in images. In short, images were stored as grey value images first and inverted. Then, a block processing command was used to find a coarse estimate of the background illumination by determining the minimum of each $5 \times 5$ pixel block in the image. The coarse estimate was then expanded in size so that it was the same size as the original image. Bicubic interpolation was used to ensure smoothness of the data. This computed illumination was subtracted from the original image to correct for the nonuniformity. The result was a darker image with minimized nonuniform illumination effects. Pixel intensities were then rescaled to fill the entire intensity range between the minimum zero and maximum one. This rescaled intensity image was thresholded with intensity values above 0.2 becoming white (value one) and below 0.2 becoming black (value zero). Areas with pixel values of 1 now corresponded to locations on the surface where neuronal tissue was present. To assure the fact that only adhesion on the background material was investigated, pixel values were adjusted to zero (black) on 140-pixel-wide bars along the length of the PEI tracks. The calculated fraction $\mathrm{F}$ of white pixels in the image now represented a quantitative measure for the adhesion on the background material only (see Fig. 1)

$$
F=\frac{\text { Number of white pixels }}{\text { Number of white pixels }+ \text { black pixels }}
$$




\section{H. Quantification of Neuron Viability}

Viability of 30-day-old neural tissue was evaluated with a staining procedure using acridine orange (AO) and propidium iodide (PPI), respectively. Stock solutions of AO (1000 $\mu \mathrm{g} / \mathrm{ml}$; Serva Feinbiochemica, Heidelberg, Germany) and PPI ( $40 \mu \mathrm{g} / \mathrm{ml}$; Sigma Aldrich, Zwijndrecht, The Netherlands) were prepared in $0.1 \mathrm{M}$ PBS. In a first run, tissue on micropatterned surfaces was stained with PPI to evaluate the presence of only nonviable tissue. Simultaneous staining of viable and nonviable tissue with both $\mathrm{AO}$ and PPI was done in a second run. In detail, each sample with adhering tissue was rinsed twice with fresh PBS and then immersed into a diluted PPI solution $(5.0 \mu \mathrm{g} / \mathrm{ml})$ for $5 \mathrm{~min}$ (first run). PPI stained (nonviable) tissuewas examined with epi-fluorescence equipment mounted on an Nikon inverted microscope Diaphot-TMD (Nikon, Japan). Photomicrographs of fluorescent images were taken with a digital photocamera (AxioCam HR, Carl Zeiss, Germany), attached to the side camera port. In the second run, the samples were immersed in a combined AO $(1.5 \mu \mathrm{g} / \mathrm{ml}) / \mathrm{PPI}$ $(10.0 \mu \mathrm{g} / \mathrm{ml})$ solution for $5 \mathrm{~min}$. The 450 - to $490-\mathrm{nm}$ excitation filter in combination with a 510-nm dichroic mirror and 520-nm barrier filter permitted the simultaneous observation of both viable (green) and nonviable (red) tissue. Photomicrographs of stained neural tissue were processed in Microsoft compatible software (Corel PhotoPaint 7, Corel Corporation, Ontario, Canada). The areas covered with red (nonviable) tissue (first run) were determined and referred to as $A_{\mathrm{NV}}$. The subsequent staining (second run) with AO and PPI resulted in images with red, green and yellow areas. Yellow is the cumulative effect of nonviable and viable cells overlapping each other, as was observed before for islets of Langerhans [26]. The surface areas where viable cells can be located is a summation of green and yellow areas and is referred to as $A_{V}$. A viability factor $V$ was defined as the ratio of the area covered with viable neurons $A_{V}$ with respect to the summation of areas covered with viable tissue $A_{V}$ and nonviable tissue $A_{\mathrm{NV}}$

$$
V=\frac{A_{V}}{A_{V}+A_{\mathrm{NV}}}
$$

\section{RESUlTS}

Fig. 2. shows bioelectrical activity originating from postnatal cortical tissue seeded onto a PEI microprinted MEA The number of spikes detected on the PEI island around electrode 34 showed an irregular patterns with a peak of approximately 35 spikes per second. The mean waveform is characterized by a fast negative peak and then followed by a slower positive peak. The morphology of the tissue is characterized by the presence of aggregates frequently positioned on top of the PEI-stamped electrodes. The background material (DDS-coated ONO) was covered with large neurite bundles interconnecting the aggregates on the PEI-stamped electrodes.

Fig. 3. shows a phase contrast image of a $15-\mu \mathrm{m}$-wide microprinted track of PEI on F127-PI. Despite the transparency of PEI, the printed borders of the tracks were visible after careful examination of the surface.

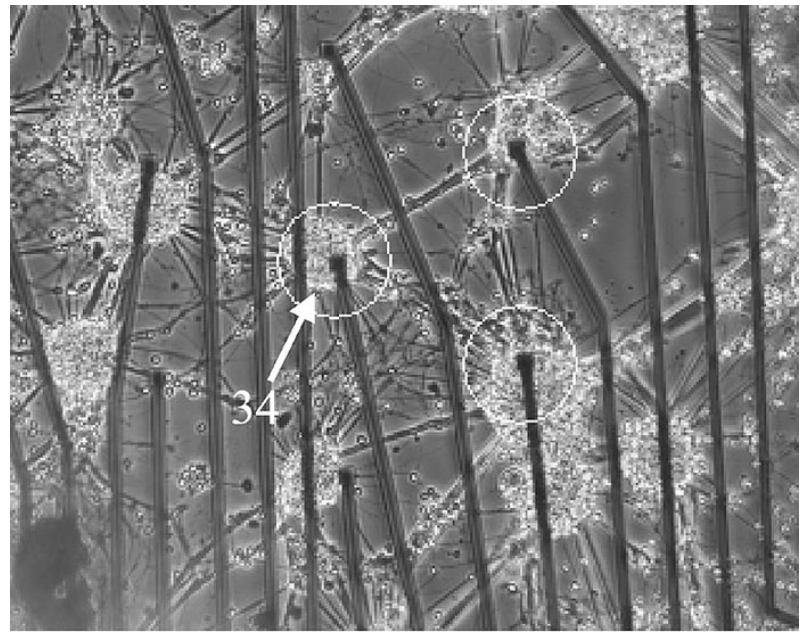

(a)

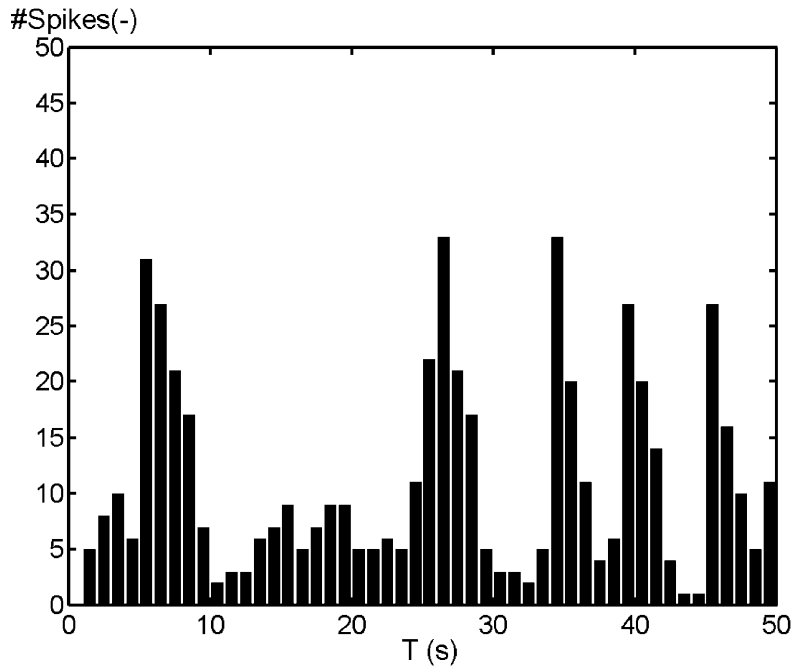

(b)

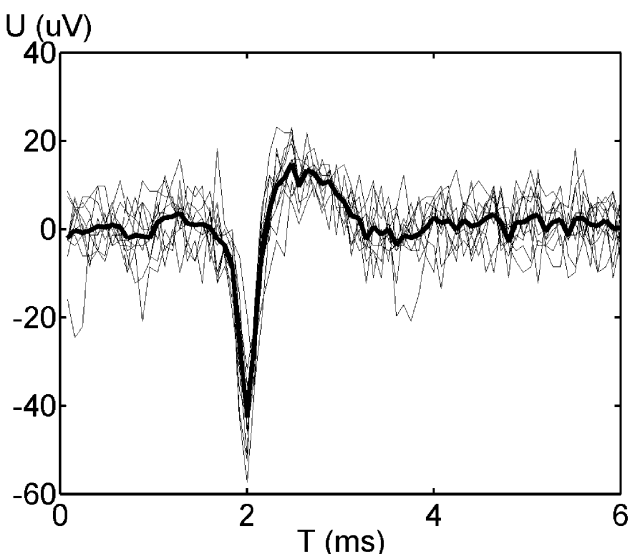

(c)

Fig. 2. Example of spontaneous bioelectrical activity from cortical neurons seeded onto a PEI-microprinted MEA (12 DIV). (a) Morphological appearance of neuronal tissue adhering on microprinted PEI circles. PEI borders are indicates by the drawn white circles. (b) Number of spikes detected on electrode number 34 in each second after onset of the measurement. The waveforms of ten spikes (electrode 34) and their mean value are presented in (c).

Fig. 4 presents the measured water contact angles on the coated samples. F108 and F127 significantly decreased contact 


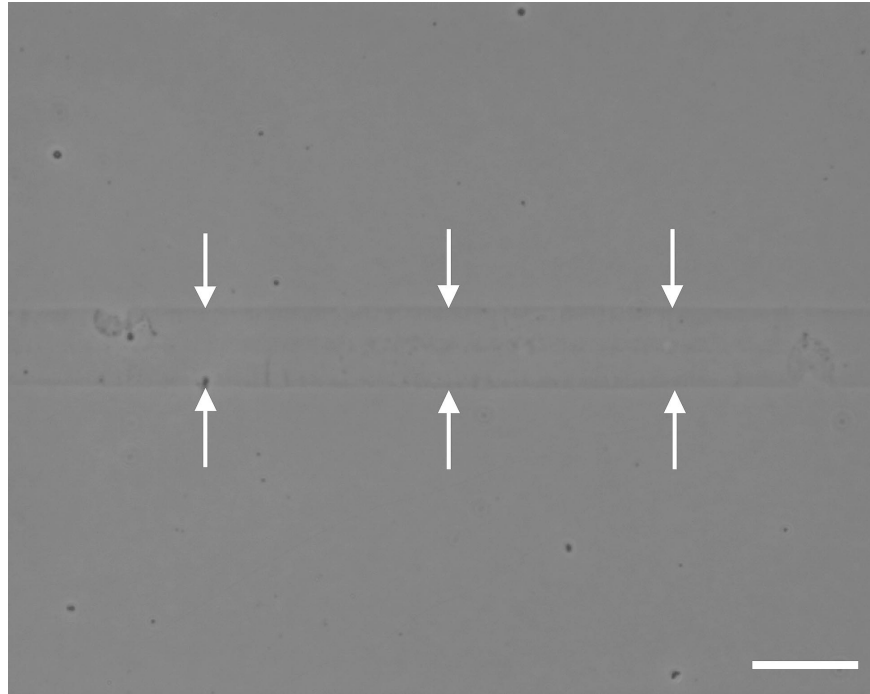

Fig. 3. Example of a microprinted track of PEI on F127-coated PI. Arrows indicate the borders of the track. Scaling bar $=20 \mu \mathrm{m}$.

Water contact angle (degrees)
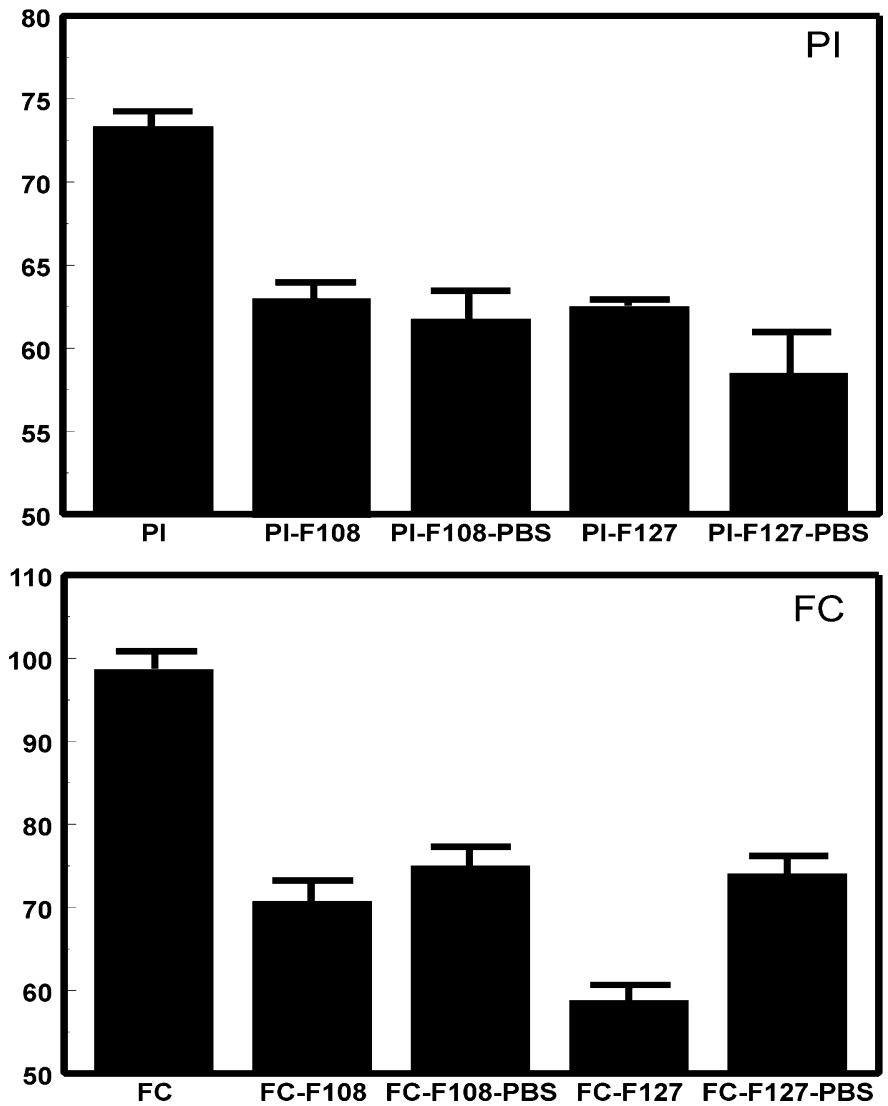

Fig. 4. Static water contact angles on F108- and F127-coated PI and FC surfaces. Data after storage in PBS for 30 days are included. Mean \pm S.D $(n=4)$

angles on both PI (initially $73^{\circ}$ ) and FC (initially $98^{\circ}$ ). The most profound effect of the F127-coating was seen on FC as the contact angle dropped from $98^{\circ}$ to $58^{\circ}$. Exposure of the coated samples to PBS (30 days) revealed no significant change on F108-PI and only a minor change on F127-PI. However, a
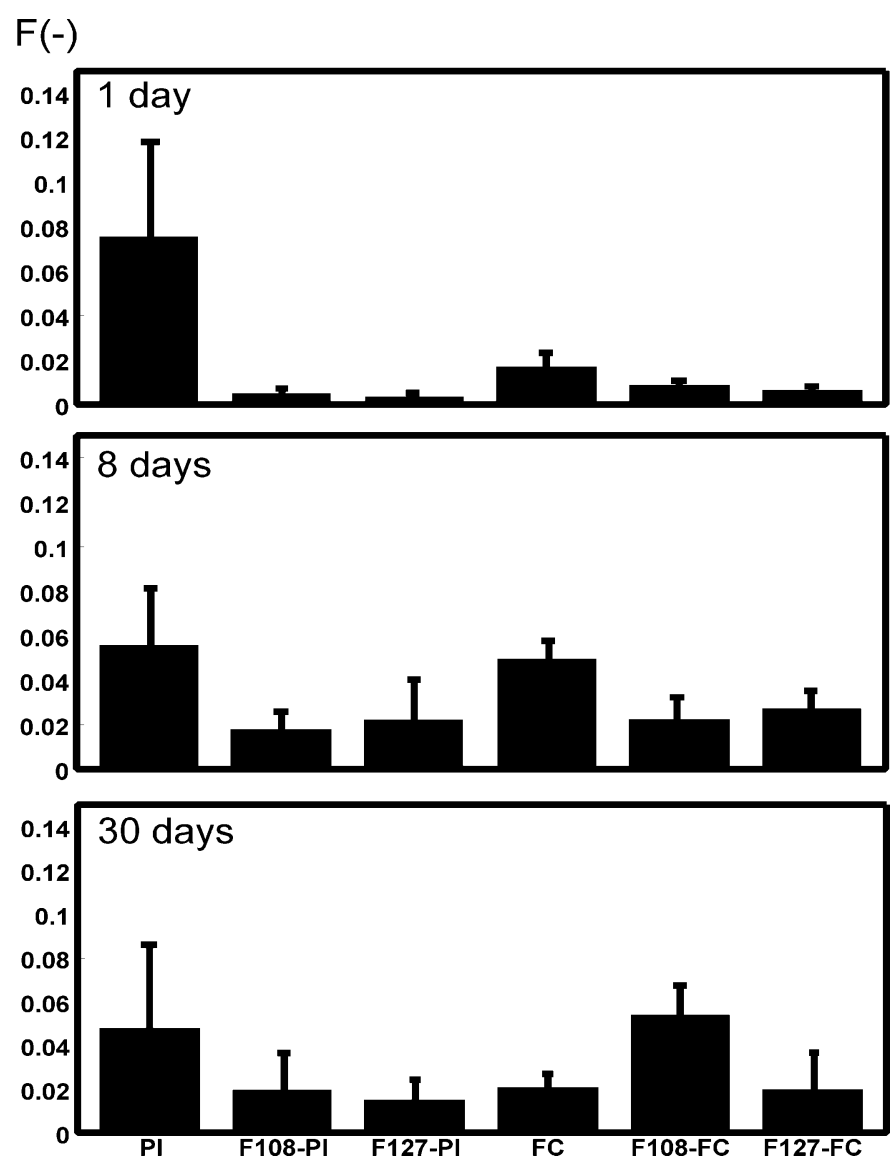

Fig. 5. Quantified background adhesion F of neuronal tissue as calculated by Matlab on different background surfaces after 1, 8, and 30 days. Mean \pm S.D $(n=6)$.

significant hydrophobic recovery was seen for F127-FC and a minor recovery for F108-FC.

Fig. 5 shows the quantified background adhesion $\mathrm{F}$ on the materials tested. Adhesion on PI was relatively high compared with the FC. The adsorbed F108 and F127 suppressed the background adhesion on FC and PI surfaces significantly after 1 day. The difference became less clear after 8 days and 30 days as background adhesion increased on the F108- and F127-coated samples.

Fig. 6 shows images of neuronal patterns on PI and F127-coated PI. After 1 day, background adhesion on PI mainly consisted of isolated cells, cell clusters, and some sparsely outgrowing neurites. The F127-coated PI was virtually free of neuronal tissue with only some isolated cells visible. The difference in neuronal adhesion onto the microprinted foreground material (PEI) and the background materials PI and F127-PI was however clear for both cases. After 30 days, the morphology of the neuronal patterns had been changed on both foreground and background surfaces. On the foreground, the relative homogeneous distribution of neurons along the PEI tracks (1 day) developed into a distribution with neurons primarily located in aggregates interconnected by neurite fascicles (30 days). The central part of the aggregates were mainly positioned on the parallel PEI tracks of the F127-coated PI as opposed to the results on PI where aggregates were also found between PEI tracks on the background surface. 


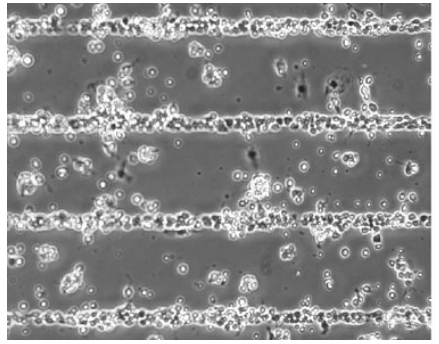

(a)

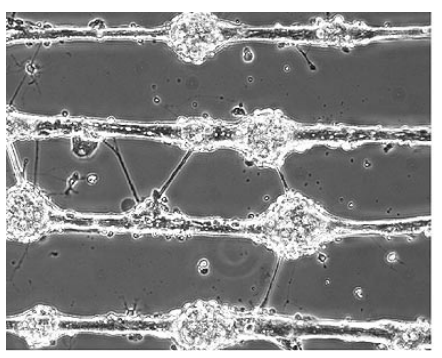

(c)

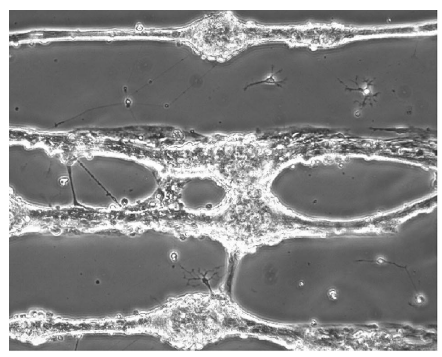

(e)

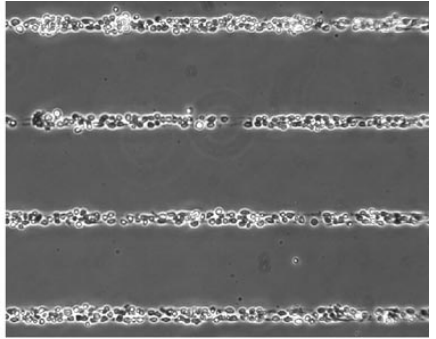

(b)

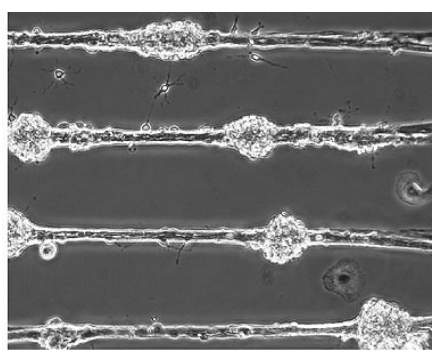

(d)

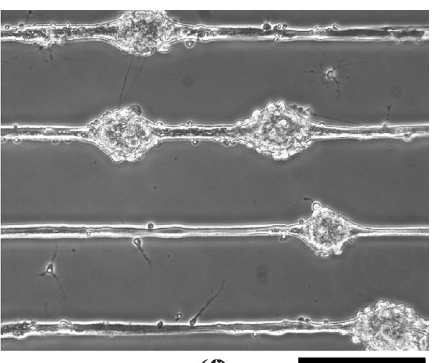

(f)
Fig. 6. Phase contrast images of adhering cortical neurons on microprinted PEI tracks after 1 day (a,b), 8 days (c,d), and 30 days (e,f). (left) Images on PI. (right) Images on F127-coated Polyimide. Scaling bar $=200 \mu \mathrm{m}$.

Fig. 7 shows the result of outgrowing neuronal tissue as observed after 30 days on a microprinted pattern of PEI circles onto FC. Cells initially migrated toward the center of the circles (not shown) and allowed free space for the outgrowth of neurites around the aggregates and within the borders of the PEI circles. The tissue on the two circles were interconnected by a bundle of neurites and/or axons.

Fig. 8 presents a representative viability stain of the neuronal tissue on the PEI-pattern on PI after 30 days. Viable and nonviable neurons co-existed on the whole pattern and especially in the aggregates. The total area covered with viable neurons outweighed the area covered with nonviable neurons resulting in a high calculated viability factor (not shown) of $0.92 \pm 0.09$ ( $n=4$. Viability on F108-PI and F127-PI was not significantly different with calculated viability factors of $0.92 \pm 0.02$ and $0.94 \pm 0.05$, respectively.

\section{DISCUSSION AND CONCLUSION}

The presence of neuronal cells in postnatal cortical tissue was demonstrated first by measurement of spontaneous bioelectrical activity on a PEI-microprinted MEA. The number of spikes detected during each time period of one second varied between zero and around 35 spikes per second [see Fig. 2(b)]. The irregularity and high number of spikes detected indicated that the measured electrical activity originated from neurons present in

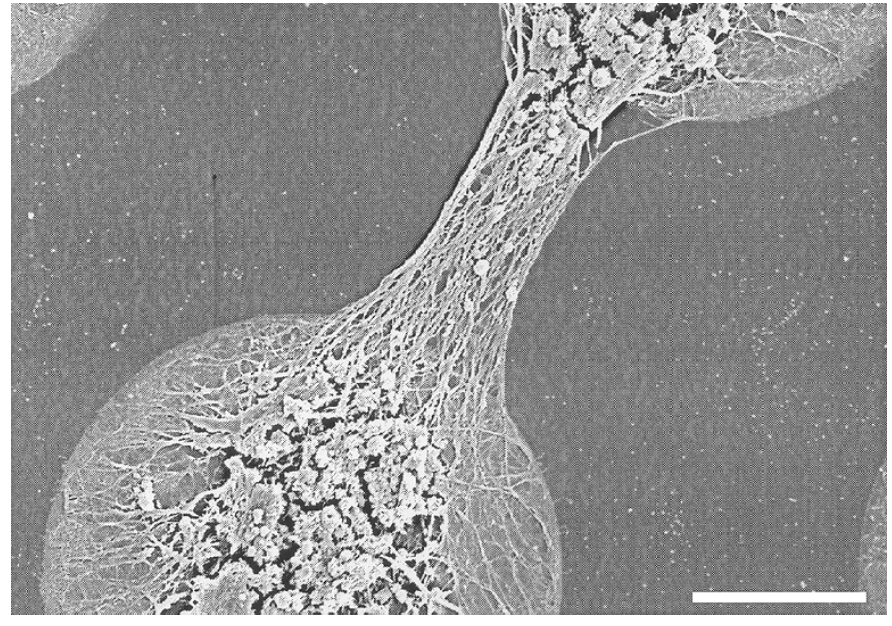

Fig. 7. Scanning electron micrograph of cortical neuronal tissue (30 days) present on a pattern of PEI circles microprinted onto FC. Diameter circles is $150 \mu \mathrm{m}$. Spacing distance between circles is $90 \mu \mathrm{m}$. Scaling bar $=50 \mu \mathrm{m}$.

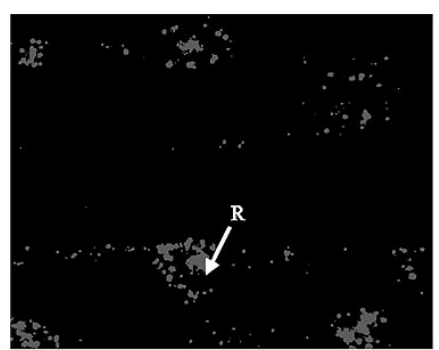

(a)

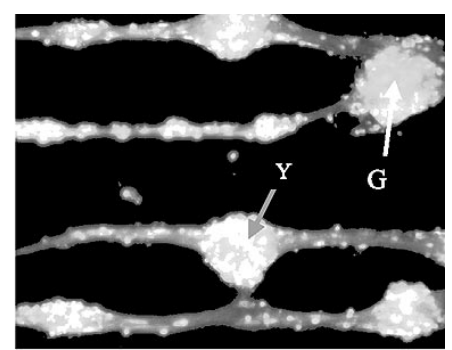

(b)
Fig. 8. Viability stain of cortical neuronal tissue (30 days) adhering on PEI tracks microprinted onto PI. Processed images of neuronal cells stained with PPI (a) and AO/PPI (b) are presented. Red (R), green (G), and yellow (Y) areas are indicated by arrows.

the postnatal tissue. In addition, the shape of the measured waveforms resembled the shape of action potentials with relatively fast rising and falling phases and slower repolarization phases [see Fig. 2(c)]. On the MEA, tissue was preferentially present on the electrodes but some tissue and especially neurite bundles interconnected aggregates over the DDS-coated ONO [see Fig. 2(a)]. The problem to maintain the geometry of patterned neuronal networks over longer periods of time critically depends upon the neuron repellent quality (read neurophobicity) of the background material [22]. Although hydrophobic surfaces like DDS-coatings are known for their ability to inhibit the adhesion of cells over short periods of time, their inability to suppress the adsorption of proteins is the critical problem that has to be overcome by adsorption of for instance PEO-PPO-PEO blockcopolymers.

One of the important findings of Nagaoka and coworkers [27] was the establishment of a relationship between the protein repulsion efficiency and PEO-chain length of covalently coupled PEO-chains. Effective protein repulsion reached a plateau level for molecular weights of approximately $5000 \mathrm{~g} / \mathrm{mol}$ [27]. In a later study, similar results were found for PEO-PPO-PEO triblockcopolymers adsorbed on hydrophobic polystyrene [28]. The applied Synperonics F108 and F127 triblockcopolymers in this study have PEO-blocks with approximate molecular weights of 5600 and $4200 \mathrm{~g} / \mathrm{mol}$ per block. Molecular weights 
of their respective hydrophobic anchoring PPO-blocks were 2800 and $3600 \mathrm{~g} / \mathrm{mol}$. It was anticipated that F127 could be less effective in preventing protein adsorption when taking into account the shorter length of the PEO-blocks but at the same time more effective because longer hydrophobic PPO-blocks interact more strongly with the hydrophobic solid substrates and, thus, hamper the possible displacement by proteins. Despite the molecular differences between both triblockcopolymers, it was demonstrated that the observed neurophobicity after 1 day was high and not significantly different for F108 and F127-coated PI and FC (Fig. 5). Apparently these opposite properties of F108 and F127 compensated each other or did not play an important role after 1 day in vitro in our experimental set-up in which a chemically defined medium R12 without serum was used [24]. The relevance of this last point was demonstrated by Caldwell et al. [29] who exposed adsorbed PEO-PPO-PEO layers to mixtures of blood plasma proteins and found replacement of PEO-PPO-PEO by these proteins on a 1-day time scale. They hypothesized that small plasma lipoproteins were responsible for the replacement of PEO-PPO-PEO and not relatively large proteins like albumin, which did not replace PEO-PPO-PEO in control experiments. As the chemically defined R12 medium in our experiments predominantly contained supplemented albumin, it could explain the efficacy of both F108 and F127-layers as neurophobic surfaces under these defined circumstances in the early culturing stage.

After 8 and 30 days in vitro neurophobicity decreased on all F108 and F127-coated surfaces. Background adhesion now was a combined effect of adhering neurons, some glial cells, outgrowing neurites and fascicles (see Fig. 6). The visibility of typical neuronal characteristics like neurites was shown in more detail with a microprinted pattern of PEI-circles. A scanning electron microscopy image of tissue connections formed between microprinted PEI circles showed that neurites and neurite fascicles were predominantly present (see Fig. 7) between PEI-circles after 30 days. On the printed PEI-track patterns, neurons were also able to penetrate onto F127-PI with growth cones originating from the PEI-tracks (Fig. 6; 8 days). A partial explanation for this could be the special ability of growth cones to explore the two-dimensional environment constantly from the PEI-tracks and to scan the local environment with dynamic filopodial extensions until an adhesive spot was found on theF127-PI background. However, it seems unlikely that filopodial extensions could easily penetrate between single PEO-chains as the average distance between chains was reported in the order of several nanometers by others [30] and, thus, significantly smaller than the average 100-nm diameter of filopodial extensions [31]. Therefore, replacement of PEO-PPO-PEO by produced endogenous proteins seems an indispensable event before adhesion of the growth tip onto the solid substrate is possible. Small proteins like the 299 amino-acid apolipoprotein $\mathrm{E}$ are the most likely candidates to replace PEO-PPO-PEO layers [32].

Comparison of the water contact angle data of the F108- and F127-coated PI and FC showed that the PI-coated surfaces were more stable during storage in PBS for 30 days (see Fig. 3). A hydrophobic recovery was observed for both F108-FC and F127-FC, which could be interpreted as an unwanted desorp- tion of the F108 and F127 from the underlying FC. Another possible explanation is chemical instability of the plasma deposited FC itself due to the presence reactive radicals in the surface and chemical restructuring of oxygen-containing groups [33]. It is, therefore, not surprising that the effect of neuron adhesion inhibition in general was more distinct on the relatively stable F108- and F127-coated PI surfaces (see Fig. 5). Another aspect is that $\mathrm{FC}$ is more hydrophobic $\left(\mathrm{WCA}=98^{\circ}\right.$ ) than PI (WCA $=73^{\circ}$ ) and already largely neurophobic through irreversibly adsorbed albumin [17]. Nevertheless, F108 and F127 were still able to inhibit the adhesion on FC more after 1 and 8 days (see Fig. 5). The ability of adsorbed F108 and F127 to inhibit not only the adhesion of growth cones but also glial cells was especially helpful for 8-day-old cultures that exhibited some glial cells adhering onto FC (not shown). This effect of F108-FC and F127-FC is not seen after 30 days because glial cells naturally detached from FC between 8 and 30 days, thus eliminating one of the most profound effects of F108 and F127 on FC. In general, both F108-PI and F127-PI displayed equal or better neurophobic background properties over 30 days and were still viable after 30-days (Fig. 8) with no observed toxic effect of the F108 and F127 affecting the tissue viability.

This study shows that the PEO-PPO-PEO coatings F108 and F127 are effective in improving the neurophobicity of hydrophobic surfaces which is essential for neuronal patterning experiments with requested high neuron-adhesive contrast between neurophilic foreground and neurophobic background. The PEO-PPO-PEO coating procedure with Synperonics F108 and F127 offers a nonlaborious means to improve the neurophobicity of MEAs with hydrophobic surfaces. The combination of the microprinted foreground material PEI with the PEO-PPO-PEO backgrounds under serum-free culturing conditions is very promising from the perspective of chemical stability of the PEI polymer chain, long-term viability of the cultured neuronal tissue on top and still relatively high pattern compliance after 30 days.

\section{ACKNOWLEDGMENT}

The authors would like to thank K.Zweers and R.Wiertz from the University of Twente for their contribution to the cell culturing experiments. The authors are greatly indebted to Prof. E. Marani of Leiden University Medical Center who continuously supported the cell culturing experiments and SEM analysis.

\section{REFERENCES}

[1] J. C. Chang, G. J. Brewer, and B. C. Wheeler, "Modulation of neural network activity by patterning," Biosens. Bioelectron., vol. 16, pp. $527-533,2001$

[2] _ - "Microelectrode array recordings of patterned hippocampal neurons for four weeks," Biomed. Microdev., vol. 2, no. 4, pp. 245-253, 2000.

[3] M. Jenkner, B. Müller, and P. Fromherz, "Interfacing a silicon chip to pairs of snail neurons connected by electrical synapses," Biological Cybern., vol. 4, pp. 239-249, 2001.

[4] P. Manos, J. J. Pancrazio, M. G. Coulombe, W. Ma, and D. A. Stenger, "Characterization of rat spinal cord neurons cultured in defined media on microelectrode arrays," Neurosci. Lett., vol. 271, pp. 179-182, 1999. 
[5] Y. Jimbo, T. Tateno, and H. P. C. Robinson, "Simultaneous induction of pathway-Specific potentiation and depression in networks of cortical neurons," Biophys. J., vol. 76, pp. 670-678, 1999.

[6] G. W. Gross and B. K. Rhoades, "The use of neuronal networks on multielectrode arrays as biosensors," Biosens. Bioelectron., vol. 10, pp. 553-567, 1995

[7] D. Kleinfeld, K. H. Kahler, and P. E. Hockberger, "Controlled outgrowth of dissociated neurons on patterned substrates," J. Neurosci., vol. 8, pp. 4098-4120, 1988.

[8] J. M. Corey, B. C. Wheeler, and G. J. Brewer, "Micrometer resolution silane-based patterning of hippocampal neurons: Critical variables in photoresist and laser ablation processes for substrate fabrication," IEEE Trans. Biomed. Eng., vol. 43, pp. 944-955, Sept. 1996.

[9] D. V. Nicolau, T. Taguchi, H. Taniguchi, H. Tanigawa, and S. Yoshikawa, "Patterning neuronal and glia cells on light-assisted functionalised photoresists," Biosens. Bioelectron., vol. 14, pp. 317-325, 1999.

[10] M. Mrksich, C. S. Chen, Y. Xia, L. E. Dike, D. E. Ingber, and G. M Whitesides, "Controlling cell attachment on contoured surfaces with self-assembled monolayers of alkanethiolates on gold," in Proc. Nat. Acad. Sci., vol. 93, 1996, pp. 10775-10778.

[11] R. Singhvi, A. Kumar, G. P. Lopez, G. N. Stephanopoulos, D. I. C. Wang G. M. Whitesides, and D. E. Ingber, "Engineering cell shape and function," Science, vol. 264, pp. 696-698, 1994.

[12] C. A. Scotchford, E. Cooper, G. J. Legget, and S. Downes, "Growth of human osteoblast-like cells on alkanethiol on gold self assembled monolayers: The effect of surface chemistry," J. Biomed. Mater. Res., vol. 41, pp. 431-442, 1998

[13] A. Folch and M. Toner, "Microengineering of cellular interactionst," Annu. Rev. Biomed. Eng., vol. 2, pp. 227-256, 2000.

[14] D. W. Branch, B. C. Wheeler, G. J. Brewer, and D. Leckband, "Long-term maintenance of patterns of hippocampal pyramidal cells on substrates of polyethylenglycol and microstamped polylysine," IEEE Trans. Biomed. Eng., vol. 47, pp. 290-300, Mar. 2000.

[15] C. K. Yeung, L. Lauer, A. Offenhauser, and W. Knoll, "Modulation of the growth and guidance of rat brain stem neurons using patterned extracellular matrix proteins," Neurosci. Lett., vol. 301, pp. 147-150, 2001

[16] A. Blau, C. Weini, J. Mach, S. Kienle, G. Jung, and C. Ziegler, "Promotion of neural cell adhesion by electrochemically generated and functionalized polymer films," J. Neurosci. Meth., vol. 112, no. 1, pp. 65-73, 2001.

[17] T. G. Ruardij, M. H. Goedbloed, and W. L. C. Rutten, "Adhesion and patterning of cortical neurons on polyethylenimine and fluorocarboncoated surfaces," IEEE Trans. Biomed. Eng., vol. 47, pp. 1593-1599, Dec. 2000.

[18] J. H. Lee, Y. M. Ju, and D. M. Kim, "Platelet adhesion onto segmented polyurethane film surfaces modified by addition and crosslinking of PEO-containing block copolymers," Biomaterials, vol. 21, pp. 683-691, 2000.

[19] G. P. Lopez, M. W. Albers, S. L. Schreiber, R. Carroll, E. Peralta, and G. M. Whitesides, "Convenient methods for patterning the adhesion of mammalian cells to surfaces using self-assembled monolayers of alkanethiolates on gold," J. Amer. Chem. Soc., vol. 115, pp. 5877-5878, 1993.

[20] M. Amiji and K. Park, "Prevention of protein adsorption and platelet adhesion on surfaces by PEO/PPO/PEO triblockcopolymers," Biomaterials, vol. 13, pp. 682-692, 1992.

[21] J. T. Li and K. D. Caldwell, "Plasma protein interactions with pluronic ${ }^{\mathrm{TM}}$-treated colloids," Colloids Surfaces B: Biointerfaces, vol. 7, pp. 9-22, 1996.

[22] D. W. Branch, B. C. Wheeler, G. J. Brewer, and D. E. Leckband, "Long-term stability of microstamped substrates of polylysine and grafted polyethylen glycol in cell culture conditions," Biomaterials, vol. 22, pp. 1035-1047, 2001

[23] R. Biran, M. D. Noble, and P. A. Tresco, "Characterization of cortical astrocytes on materials of differing surface chemistry," J. Biomed. Mater. Res., vol. 46, pp. 150-159, 1999.

[24] H. J. Romein, F. van Huizen, and P. S. Wolters, "Toward an improved serum-free, chemically defined medium for long-term culturing of cerebral cortex tissue," Neurosci. Biobehav. Rev., vol. 8, pp. 301-333, 1984.

[25] J. H. Lucas, L. E. Czisny, and G. W. Gross, "Adhesion of cultured mammalian CNS neurons to flame-modified hydrophobic surfaces," In vitro, vol. 22 , pp. $37-43,1986$.
[26] H. L. Bank, "Rapid assesment of islet viability with acridine orange and propidium iodide," In vitro Cell. Dev. Biol., vol. 24, pp. 266-273, 1988

[27] Y. Mori, S. Nagaoka, H. Takiuchi, T. Kikuchi, N. Noguchi, H. Tanzawa, and Y. Noishiki, "A new antithrombogenic material with long polyethylenoxide chains," Trans. Amer. Soc. Artif. Intern. Org., vol. 28 , pp. 459-463, 1982.

[28] J. T. Li and K. D. Caldwell, "Plasma protein interactions with pluronic TM-treated colloids," Colloids Surfaces B: Biointerfaces, vol. 7, pp. 9-22, 1996.

[29] K. D. Caldwell, "Surface modifications with adsorbed poly(ethylenoxide)-Based block copolymers," in Proc. ASC Symp. Ser. 680, J. M. Harris and S. Zalipsky, Eds., Washington, 1997, pp. 400-419.

[30] J. A. Neff, P. A. Tresco, and K. D. Caldwell, "Surface modification for controlled studies of cell-ligand interactions," Biomaterials, vol. 20, pp. 2377-2393, 1999

[31] B. Alberts, D. Bray, A. Johnson, J. Lewis, M. Raff, K. Roberts, and P. Walter, Essential Cell Biology. New York: Garland, 1998.

[32] B. P. Nathan, Y. Jiang, G. K. Wong, F. Shen, G. J. Brewer, and R. G. Struble, "Apolipoprotein E4 inhibits and apolipoprotein E3 promotes neurite outgrowth in cultured adult mouse cortical neurons through the low-density lipoprotein receptor-related protein," Brain Res., vol. 928 , pp. 96-105, 2002.

[33] H. V. Jansen, J. G. E. Gardeniers, J. Elders, H. A. C. Tilmans, and M. Elwenspoek, "Applications of fluorocarbon polymers in micromechanics and micromachining," Sensors Actuators A, vol. 41-42, pp. 136-140.

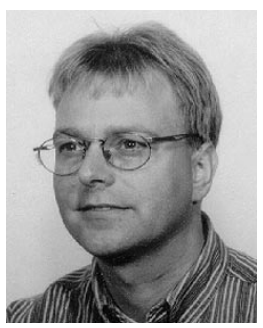

Teun G. Ruardij was born in Workum, The Netherlands, in 1967. He received the M.Sc. degree in chemical engineering from the University of Twente, Twente, The Netherlands, in 1991. His Ph.D. degree work (1993-1997) at the University of Groningen, Groningen, The Netherlands, concentrated on surface modification of biomaterials for development of vascular prosthesis.

$\mathrm{He}$ is currently a Post-Doc in the Signals \& Systems-BME group with the Department of Electrical Engineering, University of Twente. His present research interests are chemical surface modification of multielectrode arrays, neural engineering on micropatterned surfaces and development of cultured neuron probes

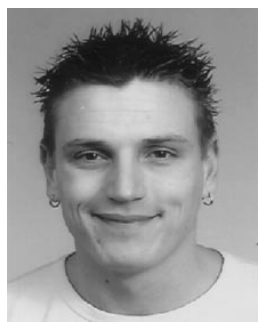

Marc A. F. van den Boogaart was born in Rotterdam, The Netherlands, in 1977. He received the B.S. degree in microengineering from the "Hogeschool van Utrecht," Utrecht, The Netherlands, in 2000. He worked on the development of thin-film microelectrode arrays, designed for interaction with cultured neurons. He is currently working towards the $\mathrm{Ph} . \mathrm{D}$. degree on a project at EPFL, Switzerland.

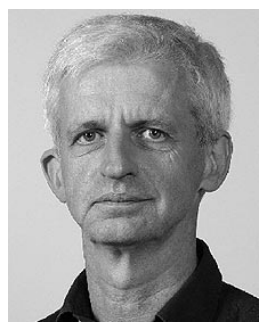

Wim L. C. Rutten (M'96) was born in 1950. He was trained as an experimental physicist and received the Ph.D. degree from Leiden University, Leiden, The Netherlands, in 1979. Thereafter, he studied the auditory system at the ENT department of Leiden University Hospital.

Since 1985, he is with BMTI/Faculty of Electrical Engineering, University of Twente, Twente, The Netherlands, as Assistant/Associate Professor of Biomedical Control Systems and Professor of Neurotechnology. His present research interests are neural engineering and cellular engineering (neuro-electronic interfaces, cultured probes), signal processing, and bioelectricity. 